\title{
The global, regional, and national burden of acute pancreatitis in 204 countries and territories, 1990-2019
}

Chang-li Li ${ }^{1}$, Meng Jiang ${ }^{2^{*}}$, Chun-qiu Pan ${ }^{3}$, Jian Li ${ }^{4}$ and Li-gang Xu ${ }^{4}$

\begin{abstract}
Background: Acute pancreatitis is a common and potentially lethal gastrointestinal disease, but literatures for the disease burden are scarce for many countries. Understanding the current burden of acute pancreatitis and the different trends across various countries is essential for formulating effective preventive intervenes. We aimed to report the incidence, mortality, and disability-adjusted life-years (DALYs) caused by acute pancreatitis in 204 countries and territories between 1990 and 2019.

Methods: Estimates from the Global Burden of Disease Study 2019 (GBD 2019) were used to analyze the epidemiology of acute pancreatitis at the global, regional, and national levels. We also reported the correlation between development status and acute pancreatitis' age-standardized DALY rates, and calculated DALYs attributable to alcohol etiology that had evidence of causation with acute pancreatitis. All of the estimates were shown as counts and agestandardized rates per 100,000 person-years.

Results: There were 2,814,972.3 (95\% UI 2,414,361.3-3,293,591.8) incident cases of acute pancreatitis occurred in 2019 globally; 1,273,955.2 (1,098,304.6-1,478,594.1) in women and 1,541,017.1 (1,307,264.4-1,814,454.3) in men. The global age-standardized incidence rate declined from 37.9/100,000 to 34.8/100,000 during 1990-2019, an annual decrease of $8.4 \%$ (5.9-10.4\%). In 2019, there were 115,053.2 (104,304.4-128,173.4) deaths and 3,641,105.7 $(3,282,952.5-4,026,948.1)$ DALYs due to acute pancreatitis. The global age-standardized mortality rate decreased by $17.2 \%$ (6.6-27.1\%) annually from 1.7/100,000 in 1990 to 1.4/100,000 in 2019; over the same period, the age-standardized DALY rate declined by $17.6 \%$ (7.8-27.0\%) annually. There were substantial differences in the incidence, mortality and DALYs across regions. Alcohol etiology attributed to a sizable fraction of acute pancreatitis-related deaths, especially in the high and high-middle SDI regions.
\end{abstract}

Conclusion: Substantial variation existed in the burden of acute pancreatitis worldwide, and the overall burden remains high with aging population. Geographically targeted considerations are needed to tailor future intervenes to relieve the burden of acute pancreatitis in specific countries, especially for Eastern Europe.

Keywords: Acute pancreatitis, Epidemiology, Disease prevention, Global burden of disease study 2019

\footnotetext{
*Correspondence: jiangmenghust@163.com

${ }^{2}$ Department of Medical Ultrasound, Tongji Hospital, Tongji Medical

College, Huazhong University of Science and Technology, Wuhan 430030,

China

Full list of author information is available at the end of the article
}

(C) The Author(s) 2021. Open Access This article is licensed under a Creative Commons Attribution 4.0 International License, which permits use, sharing, adaptation, distribution and reproduction in any medium or format, as long as you give appropriate credit to the original author(s) and the source, provide a link to the Creative Commons licence, and indicate if changes were made. The images or other third party material in this article are included in the article's Creative Commons licence, unless indicated otherwise in a credit line to the material. If material is not included in the article's Creative Commons licence and your intended use is not permitted by statutory regulation or exceeds the permitted use, you will need to obtain permission directly from the copyright holder. To view a copy of this licence, visit http://creativecommons.org/licenses/by/4.0/. The Creative Commons Public Domain Dedication waiver (http://creativeco mmons.org/publicdomain/zero/1.0/) applies to the data made available in this article, unless otherwise stated in a credit line to the data. 


\section{Background}

Acute pancreatitis is a common and potentially lethal gastrointestinal disease. Previous studies have shown a variable incidence rate, ranging from 15.0 per 100,000 in Denmark [1] to 83.7 per 100,000 in Sweden [2]. In the USA, it accounts for about 275,000 hospital admissions and $\$ 2.5$ billion health care costs each year [3]. Approximately 20\% of patients develop moderate to severe acute pancreatitis, which lead to a mortality rate of $20-40 \%$ [4-6].

The global incidence of acute pancreatitis cited in previous publications was presented as a wide range of estimates, mainly because they were based on heterogeneous study populations and varying methodological quality. In a meta-analysis, Xiao et al [7] pooled the data from general population-based cohort studies, reported that the incidence and mortality rates of acute pancreatitis were 33.7 cases and 1.6 deaths per 100,000-person years. However, the original studies included in this systematic review were confined to only five countries or territories (Sweden, Denmark, Taiwan, USA and United Kingdom), all of which were high-income regions and may not be representative of the global population. In addition, the reported incidence and mortality rates were not age-standardized, which might skew the comparisons between different regions.

Global efforts using appropriate preventive and treatment approaches to reduce the morbidity and mortality of acute pancreatitis require timely information about the burden and their risk factors. However, the current analyses on the epidemiology of acute pancreatitis were based exclusively on limited local data [1, 8-15], which inevitably subjected to selection bias and could not describe the disease burden around the globe in a robust manner. The Global Burden of Disease study (GBD), with its broad collection of data sources and the state-of-the-art statistical modelling approaches [16-18] provides us a unique opportunity to deliver the most comprehensive estimates of acute pancreatitis' burden to date. Since GBD 2017 [19], no comprehensive update of epidemiological levels and trends on acute pancreatitis have been released. In prior GBD analysis conducted by Ouyang et al. [20], the acute and chronic pancreatitis were modelled together; but in this study, we aimed to analyze acute pancreatitis separately based on GBD 2019. In this study, we summarized GBD 2019 findings on acute pancreatitis' epidemiology in 204 countries and territories, presented the temporal and geographical trends in terms of incidence, mortality, disability-adjusted life-years (DALYs), and their age-standardized rates by sex and location during 1990-2019.

\section{Methods}

\section{Data acquisition}

Acute pancreatitis in GBD 2019 were identified according to the ICD-10 code K85 and ICD-9 code 577.0. For this study, we used GBD 2019 vital registration $(19,618$ site-years of data) and verbal autopsy (374 site-years) data sources that provided a representative partial or complete sample of incidence or mortality. Information about the data sources used for each location in this study can be found on the GBD 2019 Data Input Sources Tool website (http://ghdx.healthdata.org/gbd-2019/ data-input-sources). The GBD 2019 database contains the statistical data of 369 diseases and 87 risk factors in 204 countries and territories [21,22]. The data of acute pancreatitis in GBD 2019 including incidence, mortality, DALYs, and corresponding age-standardized rates by sex and location for each year from 1990 through 2019 were obtained publicly from the Global Health Data Exchange (GHDx) website (http://ghdx.healthdata.org/gbd-resultstool). More details on the case definition, input data, data processing, and modeling strategy, as well as the differences between GBD 2019 and GBD 2017 on acute pancreatitis are provided in Additional file 1.

\section{Socio-demographic index (SDI)}

The SDI for all the 204 countries and territories during 1990-2019 was downloaded from the GHDx website for the following correlation analysis. SDI is a compound indicator of development status, created according to a country's total fertility rate for females younger than 25 years, mean education for those aged 15 years and older, and lag-distributed income per capita. Countries and territories were ranked into high, high-middle, middle, low-middle, and low SDI categories. The methods for SDI generation are detailed in previous GBD publications $[23,24]$.

\section{Statistical analysis}

The standardized methods of the GBD 2019 have been extensively reported [22]. Three main standardized tools-Cause of Death Ensemble model (CODEm), DisMod-MR 2.1 and spatiotemporal Gaussian process regression (ST-GPR) - were used to generate estimates for each quantity of interest by age, sex, location, and year. Briefly, CODEm was used to analyze death-related data. DisMod-MR 2.1 based on Bayesian meta-regression was applied to evaluate all available data on incidence and DALY. The expected relationship between SDI and age-standardized incidence, mortality and DALY rates were determined by fitting a ST-GPR for all locations from 1990 to 2019 [22]. The 95\% uncertainty intervals (UIs) were determined for each parameter using the 25th and 975th of the 1000 ordered draws based on the posterior distribution. 
The annual absolute number of incident cases, deaths, DALYs, and corresponding age-standardized rates were applied to delineate the burden of acute pancreatitis at the global, regional, and national levels. The age-standardized rates could exclude the impact from imbalance in population quantity and age distribution. DALYs was the summation of the years lived with disability (YLDs) and the years of life lost (YLLs). Moreover, the corresponding estimated annual percentage change (EAPC) values of age-standardized incidence/mortality rate (ASIR/ASMR) and age-standardized DALY rate per 100,000 people were employed to reflect the spatiotemporal trends of acute pancreatitis' burden. In the formula $Y=\alpha+\beta X, Y$ refers to $\lg$ (age-standardized rate) while $X$ means the calendar year. Then EAPC values were calculated by the formula $E A P C=100^{*}\left(10^{\beta}-1\right)$. In the case that both EAPC value and its $95 \%$ UI above zero, the corresponding agestandardized rate was in an upward trend and vice versa. Lastly, we searched the GBD 2019 database for potential risk factors that contributing to acute pancreatitis-related fatality. This study is compliant with the Guidelines for Accurate and Transparent Health Estimates Reporting (GATHER) [25].

\section{Results}

\section{Incidence of acute pancreatitis}

There were 1,727,789.3 (95\% UI 1,452,132.4-2,059,695.3) acute pancreatitis occurred in 1990 and 2,814,972.3 (95\% UI 2,414,361.3-3,293,591.8) occurred in 2019 in the globe, with an increase of $62.9 \%$ (Table 1). However, the ASIR declined by an average of $8.4 \%$ (95\% UI 5.9-10.4\%) annually during the same period, decreased from 37.9 to 34.8 per 100,000 . Males were more likely to suffer from acute pancreatitis than females (41.0 vs 34.5 per 100,000 in 1990 , and 38.8 vs 30.6 per 100,000 in 2019) (Table 1 , Fig. 1a).

In the SDI region level, the two highest quintiles of SDI regions had the highest acute pancreatitis burden in 2019, with ASIR of 38.1 and 36.7 per 100,000 respectively (Table 1 ). Subgroup analysis by geographical zone showed that Eastern Europe and High-income North America had the highest ASIR in both 1990 and 2019 (Eastern Europe: 71.2 in 1990 and 79.6 in 2019 per 100,000; High-income North America: 62.4 in 1990 and 52.0 in 2019 per 100,000$)$. Most of the regions (13/21, $61.9 \%)$ observed a steadily annual decrease in ASIR during the last 30 years. However, South Asia and Eastern Europe showed a mushrooming rise in ASIR (EAPC of South Asia: 12.8\%, 95\% UI 10.9-14.9\%; EAPC of Eastern Europe: $11.7 \%$, 95\% UI 10.8-12.7\%) (Table 1).

In 2019, countries with the greatest number of incident cases of acute pancreatitis were India $(618,862.3)$, China (493,765.4), and USA (228,699.2) (Additional file 1: Table S1). The highest ASIR (more than 60 cases per 100,000 population) were observed in the Russia $(82.0 / 100,000)$, Ukraine $(77.0 / 100,000)$, Republic of Moldova (71.3/100,000), Belarus (69.7/100,000), Slovakia $(68.4 / 100,000)$, Lithuania $(64.8 / 100,000)$, Estonia $(62.8 / 100,000)$ and Latvia $(61.7 / 100,000)$ (Fig. 2a, Additional file 1: Table S1).

\section{Mortality due to acute pancreatitis}

Globally, there were 69,817.6 (95\% UI 62,046.7-82,529.3) deaths in 1990 and 115,053.2 (95\% UI 104,304.4$128,173.4)$ deaths in 2019 caused by acute pancreatitis, increasing by $64.8 \%$ (95\% UI 55.3-68.1\%). However, the global ASMR decreased from 1.7 deaths per 100,000 (95\% UI 1.5-2.0) in 1990 to 1.4 (95\% UI 1.3-1.6) deaths per 100,000 in 2019 , with EAPC of $-17.2 \%$ (95\% UI $-27.1 \%$ to $-6.6 \%$ ) (Table 2 ). The ASMR in males was much higher than that in females ( 2.2 vs 1.3 per 100,000 in 1990; 1.9 vs 1.0 per 100,000 in 2019) (Fig. 1 b).

Subgroup analysis by SDI indicated that the highmiddle SDI region had the most deaths in both 1990 $(21,366.4)$ and $2019(34,393.0)$. As for a specific geographical zone, South Asia, Eastern Europe and East Asia were the top 3 regions with the most acute pancreatitisrelated deaths in 2019 (Table 2). India, Russia and China were the top 3 countries that had the most deaths in 2019, with 20,455.9, 11,615.3 and 10,663.6 deaths respectively (Additional file 1: Table S1). Russia also had the highest ASMR in 2019, with 5.7 (95\% UI 4.8-6.7) deaths per 100,000. Even though had a relatively large number of death cases, China had a very low ASMR in 2019, with 0.6 deaths per 100,000 and ranking eleventh from the bottom (Fig. 2b). The top five countries with the highest ASMR in 2019 were Russia $(5.7 / 100,000)$, Kazakhstan (5.0/100,000), Guinea-Bissau (4.8/100,000), Ukraine $(4.7 / 100,000)$, and Burkina Faso $(4.6 / 100,000)$ (Additional file 1: Table S1).

\section{Summary measures of health by DALYs}

The number of DALYs increased from 2,437,815.7 in 1990 to 3,641,105.7 in 2019 in the globe, but the agestandardized DALY rate improved from 59.3 in 1990 to 44.4 in 2019 per 100,000 , with EAPC of $-17.6 \%$ (95\% UI $-27.0 \%$ to $-7.8 \%$ ) (Table 3 ). Males were the main contributor to the age-standardized DALY rate compared with females (Fig. 1c). Subgroup analysis by sociodemographic factor demonstrated that although the high-middle SDI region had the most DALYs from 1990 to 2019 (720,516.0 in 1990 and 1,057,814.6 in 2019), the age-standardized DALY rate was highest in the low SDI region.

In the subgroup analysis by geographical zone, we found that Russia, Ukraine, and Republic of Moldova 
Table 1 The incidence of acute pancreatitis in 1990/2019 and temporal trends

\begin{tabular}{|c|c|c|c|c|c|}
\hline \multirow[t]{2}{*}{ Location } & \multicolumn{2}{|l|}{ Incident cases (95\% UI) } & \multicolumn{2}{|c|}{$\begin{array}{l}\text { Age-standardized incidence } \\
\text { rate (per 100,000) }\end{array}$} & \multirow{2}{*}{$\begin{array}{l}\text { EAPC of incidence rate } \\
1990-2019(\%)\end{array}$} \\
\hline & 1990 & 2019 & 1990 & 2019 & \\
\hline Global & $\begin{array}{l}1,727,789.3(1,452,132.4- \\
2,059,695.3)\end{array}$ & $\begin{array}{l}2,814,972.3(2,414,361.3- \\
3,293,591.8)\end{array}$ & $37.9(32-44.6)$ & $34.8(29.8-40.7)$ & $-8.4(-10.4$ to -5.9$)$ \\
\hline \multicolumn{6}{|l|}{ Sex } \\
\hline Male & $\begin{array}{l}929,028.7(774,587.6- \\
\quad 1,115,575.6)\end{array}$ & $\begin{array}{l}1,541,017.1(1,307,264.4- \\
1,814,454.3)\end{array}$ & $41.0(34.3-48.5)$ & $38.8(33.1-45.5)$ & $-5.4(-7.5$ to -2.8$)$ \\
\hline Female & $\begin{array}{l}798,760.6(677,669.1- \\
943,983.6)\end{array}$ & $\begin{array}{l}1,273,955.2(1,098,304.6- \\
1,478,594.1)\end{array}$ & $34.5(29.2-40.3)$ & $30.6(26.4-35.6)$ & $-11.2(-13.2$ to -8.8$)$ \\
\hline \multicolumn{6}{|l|}{ SDI factor } \\
\hline High SDI & $\begin{array}{l}390,419.1(335,676.7- \\
452,156.8)\end{array}$ & $\begin{array}{l}533,633.2(477,729.8- \\
597,075.3)\end{array}$ & $41.3(35.4-48.0)$ & $38.1(33.9-42.7)$ & $-7.9(-11.8$ to -3.1$)$ \\
\hline High-middle SDI & $\begin{array}{l}482,857.1(406,521.4- \\
568,658.8)\end{array}$ & $\begin{array}{l}667,222.6(569,618.5- \\
\quad 769,408.6)\end{array}$ & $43.2(36.6-50.6)$ & $36.7(31.5-42.6)$ & $-15.0(-16.6$ to -13.1$)$ \\
\hline Middle SDI & $\begin{array}{l}455,054.8(373,090.6- \\
554,469.3)\end{array}$ & $\begin{array}{l}735,082.3(616,164.7- \\
867,454.5)\end{array}$ & $33.7(27.8-40.1)$ & $28.8(24.4-34.2)$ & $-14.3(-16.7$ to -11.4$)$ \\
\hline Low-middle SDI & $\begin{array}{l}297,240.1(244,736.5- \\
361,676.3)\end{array}$ & $\begin{array}{l}598,531.4(497,030.5- \\
720,521.9)\end{array}$ & $34.9(29.0-41.8)$ & $36.5(30.7-43.7)$ & $4.6(3.0-6.4)$ \\
\hline Low SDI & $101,572(83,136.7-123,390.1)$ & $\begin{array}{l}224,224.5(183,906.9- \\
270,951.5)\end{array}$ & $28.4(23.8-33.7)$ & $27.7(23.4-32.9)$ & $-2.4(-3.5$ to -1.3$)$ \\
\hline \multicolumn{6}{|l|}{ Region } \\
\hline Andean Latin America & $12,751.6(10,888.9-14,964.7)$ & $26,446.1(23,242.3-30,101.7)$ & $45.8(39.6-52.9)$ & $43.5(38.4-49.3)$ & $-5.0(-7.3$ to -2.6$)$ \\
\hline Australasia & $8618.4(7250.0-10,167.5)$ & $14,394.8(12,298.7-16,770.4)$ & $38.6(32.5-45.3)$ & $37.6(31.7-44)$ & $-2.8(-5.2$ to -0.3$)$ \\
\hline Caribbean & $8807.7(7294.7-10,668.9)$ & $14,155.4(11,772.9-16,692.7)$ & $28.8(23.9-34.3)$ & $28.4(23.5-33.6)$ & $-1.7(-2.9$ to -0.4$)$ \\
\hline Central Asia & $18,365.6(15,467.5-21,700.0)$ & $29,035.3(24,076.9-34,326.1)$ & $33.7(28.4-39.4)$ & $32.9(27.7-38.5)$ & $-2.4(-3.4$ to -1.1$)$ \\
\hline Central Europe & $67,402.6(57,280.6-78,292.3)$ & $73,015.8(64,542.3-82,156.6)$ & $49.4(42-57.3)$ & $45.2(40.1-50.9)$ & $-8.6(-12.5$ to -3.2$)$ \\
\hline Central Latin America & $46,647.8(39,437.0-55,591.9)$ & $\begin{array}{l}96,777.3(83,302.4- \\
\quad 112,767.0)\end{array}$ & $37.6(32.1-44)$ & $38.6(33.3-44.7)$ & $2.6(1.0-4.4)$ \\
\hline Central Sub-Saharan Africa & 7427.2 (6052.5-9165.5) & $18,256.3(14,929.3-22,374.1)$ & $21.1(17.6-25)$ & $20.8(17.5-24.7)$ & $-1.0(-2.9$ to -0.9$)$ \\
\hline East Asia & $\begin{array}{l}396,687.7(322,719.1- \\
\quad 482,300.5)\end{array}$ & $\begin{array}{l}526,066.5(444,786.6- \\
615,193.3)\end{array}$ & $38(31.1-45.4)$ & $27.6(23.5-32.2)$ & $-27.4(-30.4$ to -23.8$)$ \\
\hline Eastern Europe & $\begin{array}{l}182,577.6(155,056.7- \\
211,692.2)\end{array}$ & $\begin{array}{l}221,945.2(188,142.5- \\
258,013.8)\end{array}$ & $71.2(60.8-82.9)$ & $79.6(68.2-92.5)$ & $11.7(10.8-12.7)$ \\
\hline Eastern Sub-Saharan Africa & $25,232.9(20,531.3-31,140.5)$ & $57,965.7(46,838.7-71,275.5)$ & $21.1(17.7-25.2)$ & $21.1(17.7-25.2)$ & $0.1(-0.6$ to -0.7$)$ \\
\hline High-income Asia Pacific & $62,652.3(52,382.8-74,360.9)$ & $78,997.7(68,905.1-90,260.6)$ & $32.9(27.6-39.1)$ & $31.5(27.3-36.5)$ & $-4.3(-9.0$ to -1.3$)$ \\
\hline $\begin{array}{l}\text { High-income North } \\
\text { America }\end{array}$ & $\begin{array}{l}200,836.4(173,238.5- \\
232,436.8)\end{array}$ & $\begin{array}{l}257,777.8(236,252.7- \\
284,187.5)\end{array}$ & $62.4(53.7-72.0)$ & $52.0(47.5-56.9)$ & $-16.6(-22.3$ to -9.9$)$ \\
\hline $\begin{array}{l}\text { North Africa and Middle } \\
\text { East }\end{array}$ & $61,824.3(50,782.1-75,020.1)$ & $\begin{array}{l}140,637.9(117,038.5- \\
168,090.2)\end{array}$ & $26.7(22.3-31.5)$ & $26.6(22.5-31.2)$ & $-0.2(-1.7$ to -1.6$)$ \\
\hline Oceania & $1078.6(884.9-1322.0)$ & $2385.6(1934.6-2925.5)$ & $24.9(20.7-29.6)$ & $24.1(20-28.7)$ & $-3.0(-5.0$ to -0.8$)$ \\
\hline South Asia & $\begin{array}{l}328,618.8(267,223.4- \\
\quad 401,086.7)\end{array}$ & $\begin{array}{l}\text { 743,524.0 (611,176.0- } \\
901,906.4)\end{array}$ & $38.1(31.6-45.9)$ & $43.0(35.9-51.7)$ & $12.8(10.9-14.9)$ \\
\hline Southeast Asia & $\begin{array}{l}93,540.7(76,473.3- \\
113,689.3)\end{array}$ & $\begin{array}{l}174,246.5(143,846.9- \\
208,675.3)\end{array}$ & $26.0(21.6-30.9)$ & $25.3(21.2-30.1)$ & $-2.6(-3.9$ to -1.5$)$ \\
\hline Southern Latin America & $15,849.3(13,664.8-18,265.2)$ & $24,167.2(20,962.5-27,835.3)$ & $33.6(28.9-38.6)$ & $31.6(27.4-36.6)$ & $-5.8(-7.9$ to -3.9$)$ \\
\hline $\begin{array}{l}\text { Southern Sub-Saharan } \\
\text { Africa }\end{array}$ & $8604.9(7042.0-10,531.2)$ & $15,207.3(12,560.8-18,528.5)$ & $22.1(18.5-26.4)$ & $21.7(18.1-25.9)$ & $-5.8(-7.9$ to -3.9$)$ \\
\hline Tropical Latin America & $23,704.1(20,682.2-27,345.5)$ & $47,509.1(41,862.2-53,770.0)$ & $20.1(17.7-22.7)$ & $19.4(17.1-21.9)$ & $-3.4(-4.7$ to -2.0$)$ \\
\hline Western Europe & $\begin{array}{l}123,270.9(107,562.6- \\
140,276.4)\end{array}$ & $\begin{array}{l}170,344.2(149,047.3- \\
194,756.8)\end{array}$ & $25.4(22.1-29.2)$ & $26.3(22.8-30.1)$ & $3.5(1.5-5.3)$ \\
\hline $\begin{array}{l}\text { Western Sub-Saharan } \\
\text { Africa }\end{array}$ & $33,289.8(27,664.2-40,190.6)$ & $82,116.6(68,165.6-98,901.6)$ & $26.0(22.0-30.6)$ & $26.7(22.7-31.4)$ & $3.0(2.5-3.5)$ \\
\hline
\end{tabular}



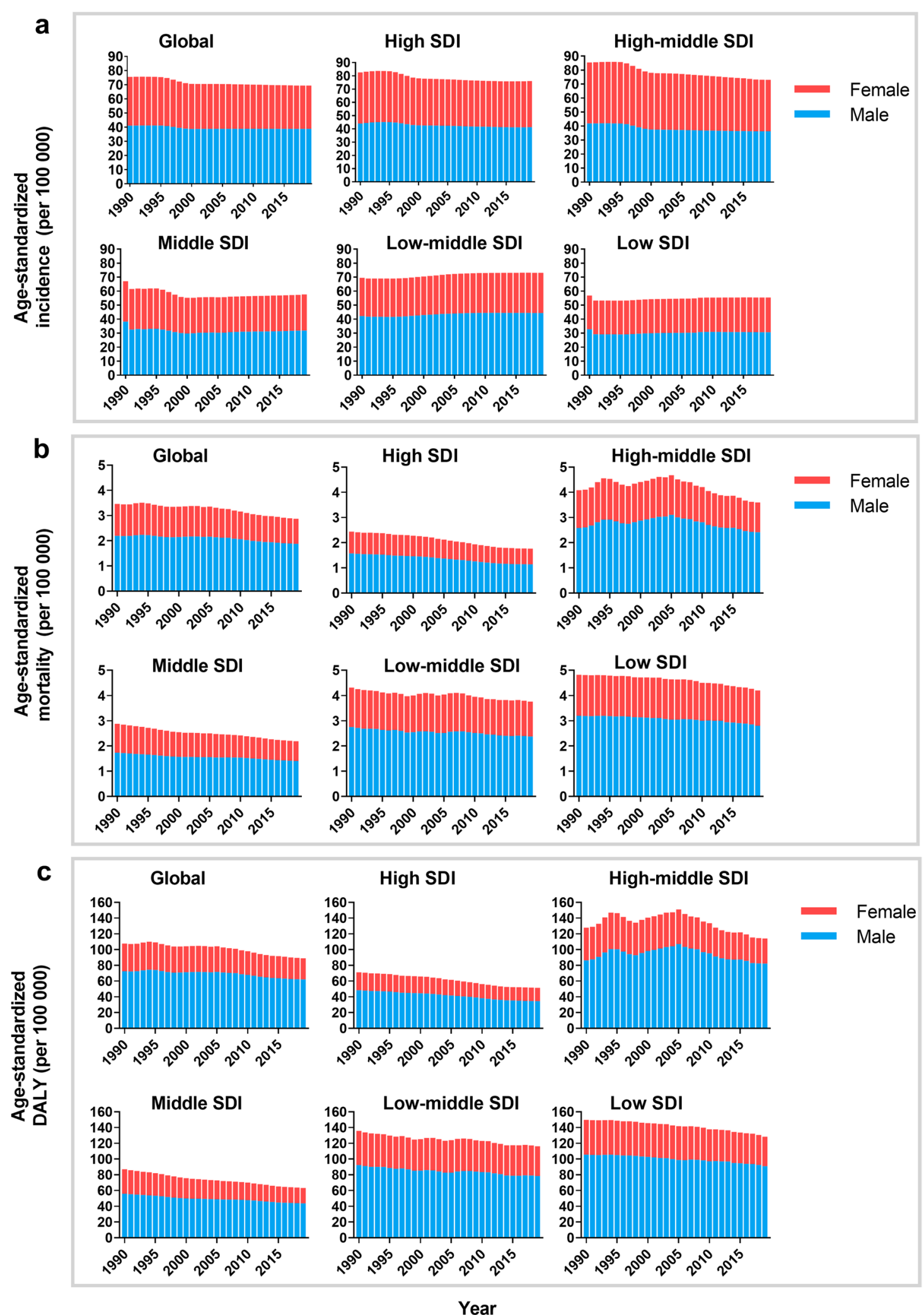

Fig. 1 The change trends of age-standardized acute pancreatitis' incidence (a), mortality (b), and DALY rates (c) per 100,000 person-years from 1990 to 2019. DALY: disability-adjusted life-year; SDI: social demographic index 
a
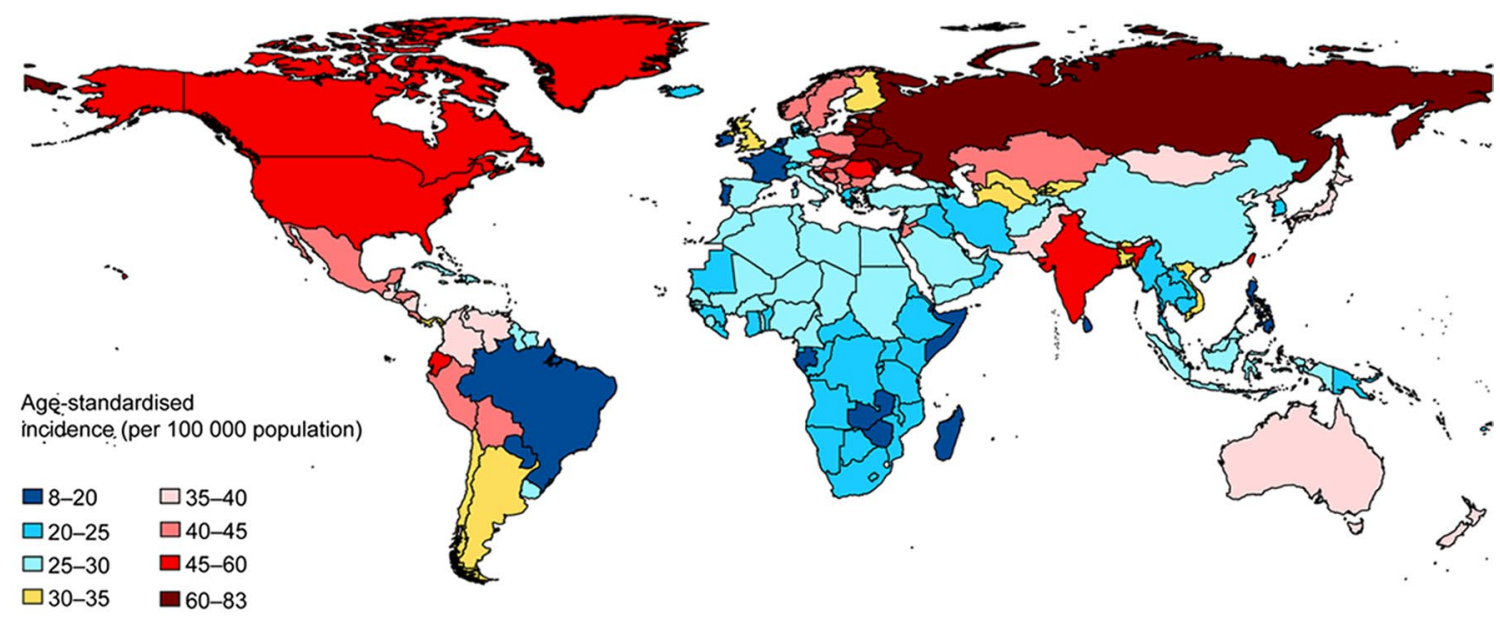

b
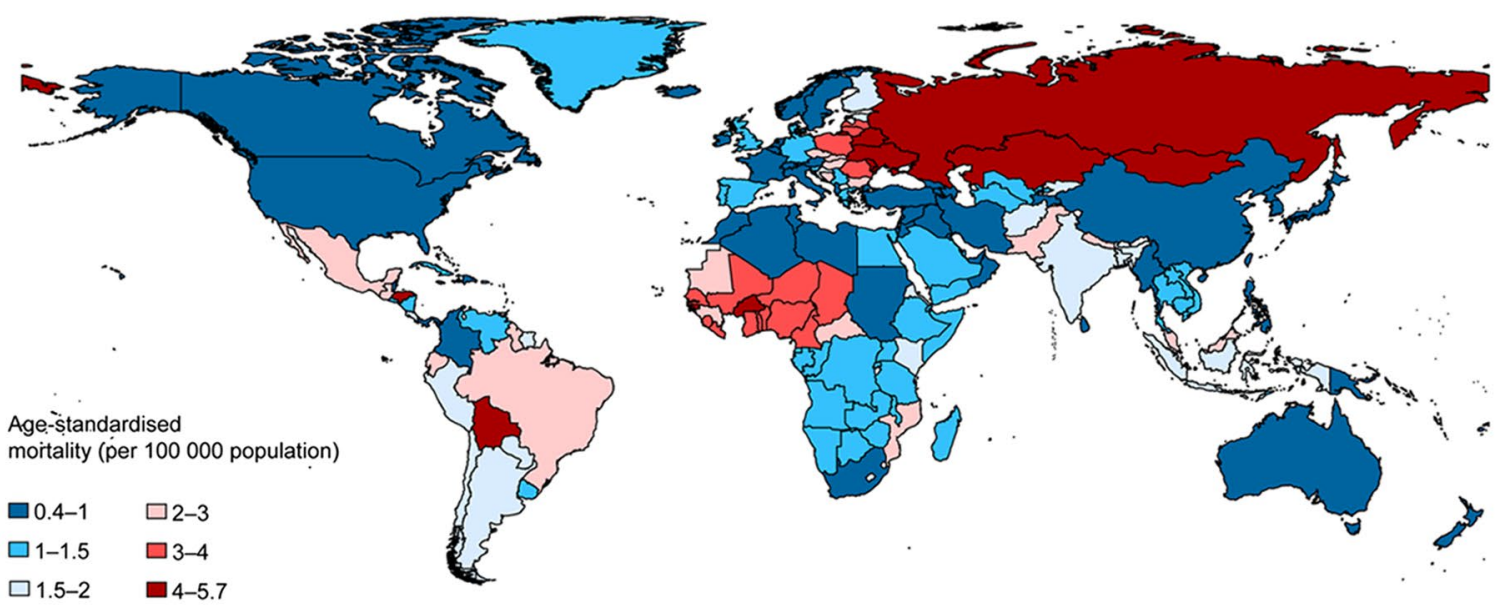

C

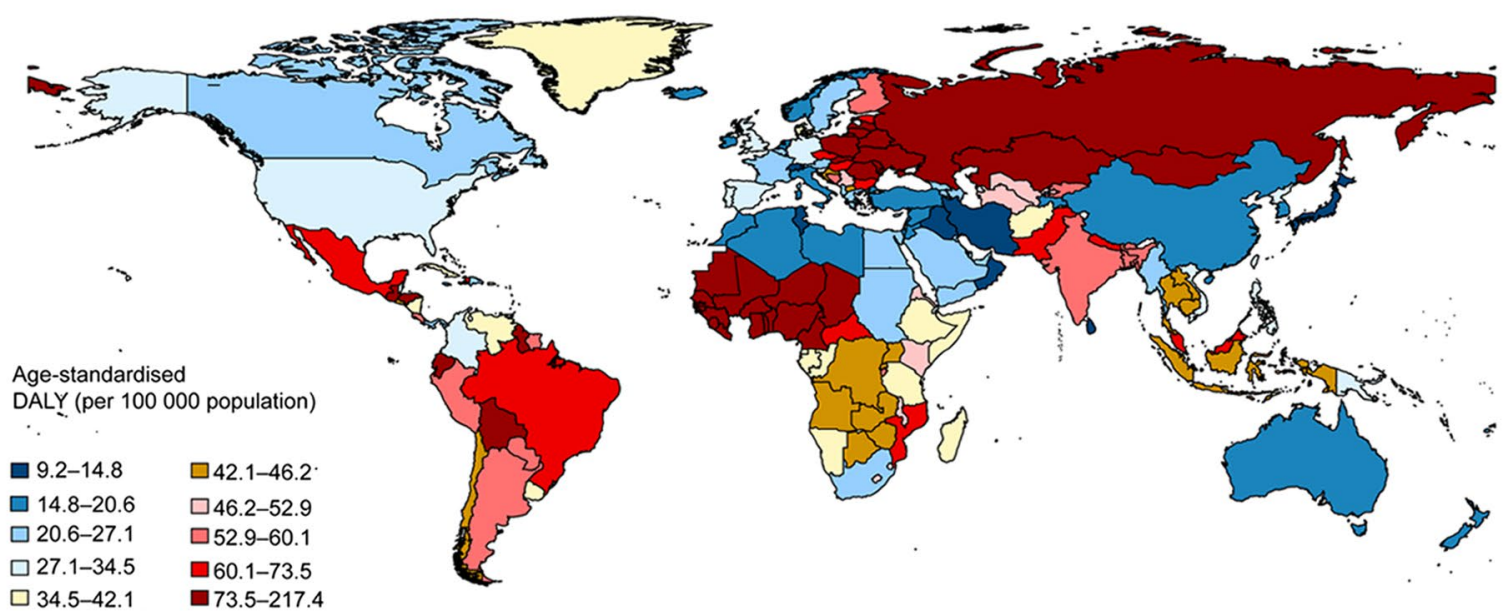

Fig. 2 The age-standardized incidence (a), mortality (b) and DALY rates (c) of acute pancreatitis per 100,000 person-years by location for both sexes combined, 2019. DALY: disability-adjusted life-year 
Table 2 The death of acute pancreatitis in 1990/2019 and temporal trends

\begin{tabular}{|c|c|c|c|c|c|}
\hline & \multicolumn{2}{|l|}{ Death cases (95\% UI) } & \multicolumn{2}{|c|}{$\begin{array}{l}\text { Age-standard mortality } \\
\text { rate (per 100,000) }\end{array}$} & \multirow{2}{*}{$\begin{array}{l}\text { EAPC of mortality rate } \\
1990-2019(\%)\end{array}$} \\
\hline & 1990 & 2019 & 1990 & 2019 & \\
\hline Global & $69,817.6(62,046.7-82,529.3)$ & $115,053.2(104,304.4-128,173.4)$ & $1.7(1.5-2.0)$ & $1.4(1.3-1.6)$ & $-17.2(-27.1$ to -6.6$)$ \\
\hline \multicolumn{6}{|c|}{ 为 } \\
\hline Male & $43,129.0(37,615.8-51,561.2)$ & $71,983.2(63,882.3-81,418.6)$ & $2.2(1.9-2.6)$ & $1.9(1.7-2.1)$ & $-14.5(-24.4$ to -3.1$)$ \\
\hline Female & $26,688.6(23,528.9-32,952.8)$ & $43,070(36,592.6-50,773.7)$ & $1.3(1.1-1.6)$ & $1.0(0.8-1.2)$ & $-21.5(-36.2$ to -8.3$)$ \\
\hline \multicolumn{6}{|l|}{ SDI factor } \\
\hline High SDI & $12,159.0(11,441.0-13,388.5)$ & $16,160.0(14,600.9-18,476.3)$ & $1.2(1.1-1.3)$ & $0.9(0.8-1.0)$ & $-26.9(-30.9$ to -21.5$)$ \\
\hline High-middle SDI & $21,366.4(19,824.1-25,093.1)$ & $34,393.0(31,194.4-37,307.4)$ & $2.0(1.9-2.4)$ & $1.8(1.6-1.9)$ & $-11.7(-27.4$ to -3.0$)$ \\
\hline Middle SDI & $15,561.3(13,213.7-20,288.1)$ & $25,776.2(22,835.9-30,111.2)$ & $1.4(1.2-1.9)$ & $1.1(1.0-1.3)$ & $-24.7(-39.6$ to -7.6$)$ \\
\hline Low-middle SDI & $14,314.8(11,855.7-18,507.1)$ & $26,440.8(21,686.3-30,710.8)$ & $2.2(1.8-2.8)$ & $1.9(1.5-2.2)$ & $-13.7(-31.8$ to -13.1$)$ \\
\hline Low SDI & $6385.7(4653.4-8612.9)$ & $12,232.2(9722.6-15,560.5)$ & $2.4(1.7-3.3)$ & $2.1(1.7-2.7)$ & $-13.5(-27.8$ to -4.8$)$ \\
\hline \multicolumn{6}{|l|}{ Region } \\
\hline Andean Latin America & $1076.8(811.2-1299.4)$ & $1444.9(1122.3-1971.2)$ & $4.6(3.4-5.6)$ & $2.5(2.0-3.5)$ & $-44.2(-59.6$ to -15.1$)$ \\
\hline Australasia & $215.9(198.6-233.7)$ & $339(292.5-396.5)$ & $0.9(0.9-1)$ & $0.7(0.6-0.8)$ & $-29.7(-37.7$ to -17.5$)$ \\
\hline Caribbean & $384.3(338.7-436.9)$ & 659.0(557.6-795.7) & $1.4(1.3-1.6)$ & $1.3(1.1-1.6)$ & $-9.6(-24.2$ to 7.0$)$ \\
\hline Central Asia & $1179.1(1016.3-1321.5)$ & $1653.3(1381.5-1884.1)$ & $2.4(2.1-2.8)$ & $2.1(1.8-2.4)$ & -12.1 ( -24.3 to 1.7$)$ \\
\hline Central Europe & $4476.7(4274.1-5033.9)$ & $5140.4(4513.4-5828.0)$ & $3.2(3.1-3.6)$ & $2.7(2.4-3.1)$ & $-15.6(-27.2$ to -4.2$)$ \\
\hline Central Latin America & $2018.5(1909.5-2130.6)$ & $4332.6(3728.8-5065.2)$ & $2.1(1.9-2.2)$ & $1.8(1.6-2.1)$ & $-12.4(-24.8$ to2.2) \\
\hline Central Sub-Saharan Africa & $452.4(302.2-763.6)$ & $911.5(530.4-1648.7)$ & $1.8(1.2-3.1)$ & $1.4(0.8-2.7)$ & - 19.7 (v43 to 4.6) \\
\hline East Asia & $9323.9(7503.7-12,418.1)$ & 11,289.8 (8798.1-13,450.7) & $1.1(0.9-1.5)$ & $0.6(0.5-0.7)$ & $-44.3(-60.6$ to -26.3$)$ \\
\hline Eastern Europe & $7662.1(6921.7-10,789)$ & $15,578.4(13,366.9-17,734.7)$ & $2.9(2.6-4.1)$ & $5.3(4.5-6)$ & 83.2(20.1-115.4) \\
\hline Eastern Sub-Saharan Africa & 1398.2(899.1-2104.9) & $2769.3(1710.4-4739.2)$ & $1.7(1.0-2.6)$ & $1.5(0.9-2.7)$ & -12.1 ( -34.6 to 9.5$)$ \\
\hline High-income Asia Pacific & $1792.4(1602.4-2057.1)$ & $2248.0(1886.8-2803.8)$ & $1.0(0.9-1.1)$ & $0.5(0.4-0.6)$ & $-49.5(-55.7$ to -39.5$)$ \\
\hline High-income North America & $3348.2(3123.7-3579.4)$ & $5443.7(4993.9-5955.3)$ & $1.0(0.9-1)$ & $0.9(0.9-1.0)$ & $-5.6(-9.6$ to 1.3$)$ \\
\hline North Africa and Middle East & $1718.7(1389-2350.2)$ & $3394.9(2654.5-4069.2)$ & $1.1(0.9-1.5)$ & $0.9(0.7 .0-1.1)$ & $-21.2(-42.6$ to 2.9$)$ \\
\hline Oceania & $36.8(25.7-52.2)$ & $76.7(53.7-107.9)$ & $1.1(0.7-1.5)$ & $0.9(0.7-1.3)$ & $-11.9(-32$ to 11$)$ \\
\hline South Asia & $14,050.3(11,344.3-19,215)$ & $25,936.8(20,085.4-31,351.7)$ & $2.3(1.8-3.1)$ & $1.8(1.4-2.2)$ & $-20.2(-42.4$ to 11.9$)$ \\
\hline Southeast Asia & $4993.4(3842.9-7457.8)$ & $7913.5(6540.7-11,170.8)$ & $1.8(1.4-2.6)$ & $1.3(1.1-1.8)$ & $-25.1(-40.9$ to -2.5$)$ \\
\hline Southern Latin America & $1267.6(1136.0-1374.1)$ & $1495.7(1347.0-1732.5)$ & $2.8(2.5-3.0)$ & $1.8(1.7-2.1)$ & $-33.8(-42.5$ to -17.9$)$ \\
\hline Southern Sub-Saharan Africa & $325.4(263.3-414.7)$ & $573.9(453-675.4)$ & $1.0(0.8-1.3)$ & $0.9(0.7-1.1)$ & -7.8 (- 33.5 to 10.6$)$ \\
\hline Tropical Latin America & 2329.6(2212.2-2485.9) & $5557.4(4793.8-5987.2)$ & $2.3(2.1-2.4)$ & $2.3(2.0-2.5)$ & $1.0(-15.1$ to 12$)$ \\
\hline Western Europe & 7986.7 (7447.9-8873.5) & $9984.5(8925.7-11,455.5)$ & $1.4(1.4-1.6)$ & $1.1(1.0-1.2)$ & $-25.7(-30.9$ to -19.4$)$ \\
\hline Western Sub-Saharan Africa & $3781.0(2498.4-5769.9)$ & $8310.1(5927.1-11,883.0)$ & $3.8(2.5-5.8)$ & $3.6(2.7-5.1)$ & $-3.9(-30.4$ to 28.5$)$ \\
\hline
\end{tabular}

EAPC, estimated annual percentage change; UI, uncertainty interval

were the top 3 countries that had the highest age-standardized DALY rate in 2019 (with 217.3, 196.2 and 173.1 per 100,000 respectively) (Fig. 2c). Republic of Korea had the fastest decrease in age standard DALY rate during the past 30 years (dropped from 47.3 to 17.7 per 100,000, $\mathrm{EAPC}=-62.6 \%, 95 \%$ UI $-79.9 \%$ to $-33.6 \%)$. By contrast, Russia showed a sharply increase in age-standardized DALY rate from 1990 to 2019, increased from 103.1 to 217.3 per 100,000 (EAPC $=110.9 \%$, 95\% UI $26.3-$ 155.3\%) (Additional file 1: Table S1).

\section{The correlation between SDI and the burden of acute} pancreatitis

We investigated the correlation between SDI and ASIR, ASMR, and age-standardized DALY rate in 21 regions around the globe from 1990 to 2019. The results revealed that the ASIR was positively correlated with SDI $(P<0.001)$. In the contrary, both the ASMR $(P=0.001)$ and age-standardized DALY rate $(P=0.037)$ were negatively correlated with SDI (Fig. 3). Notably, despite gains in SDI over time, Eastern Europe had much higher agestandardized incidence, mortality and DALY rates than 
Table 3 The DALYs of acute pancreatitis in 1990/2019 and temporal trends

\begin{tabular}{|c|c|c|c|c|c|}
\hline & \multicolumn{2}{|l|}{ DALYs (95\% UI) } & \multicolumn{2}{|c|}{$\begin{array}{l}\text { Age-standardized DALY rate (per } \\
100,000)\end{array}$} & \multirow{2}{*}{$\begin{array}{l}\text { EAPC of DALY } \\
1990-2019(\%)\end{array}$} \\
\hline & 1990 & 2019 & 1990 & 2019 & \\
\hline Global & $\begin{array}{l}2,437,815.7(2,179,992.9- \\
2,885,021.2)\end{array}$ & $\begin{array}{r}3,641,105.7(3,282,952.5- \\
4,026,948.1)\end{array}$ & $53.9(48.2-63.3)$ & $44.4(40.1-49.1)$ & $-17.6(-27.0$ to -7.8$)$ \\
\hline Male & $\begin{array}{l}1,633,012.8(1,426,941.6- \\
1,942,851.5)\end{array}$ & $\begin{array}{r}2,502,438.2(2,224,864.1- \\
842,383.8)\end{array}$ & $72.4(63.4-85.9)$ & $62.0(55.2-70.4)$ & $-14.4(-23.8$ to -3.4$)$ \\
\hline Female & $\begin{array}{l}804,802.9(691,735.0- \\
1,038,584.8)\end{array}$ & $\begin{array}{l}1,138,667.5(969,571.1- \\
1,334,422.8)\end{array}$ & $35.2(30.6-44.8)$ & $27.0(22.9-31.5)$ & $-23.5(-38.2$ to -11.9$)$ \\
\hline \multicolumn{6}{|l|}{ SDI factor } \\
\hline High SDI & $\begin{array}{l}341,420.8(321,211.0- \\
370,387.3)\end{array}$ & - $389,932.5(360,177.7-$ & $35.3(33.2-38.3)$ & $25.7(23.8-28.4)$ & $-27.1(-31.8$ to -22.7$)$ \\
\hline High-middle SDI & $\begin{array}{l}720,516.0(667,787.2- \\
839,145.4)\end{array}$ & $\begin{array}{l}1,057,814.6(962,196.3- \\
1,156,821.3)\end{array}$ & $63.7(59.1-74.2)$ & $57.1(51.9-62.4)$ & $-10.5(-24.7$ to -1.7$)$ \\
\hline Middle SDI & $\begin{array}{l}580,739.7(498,484.7- \\
739,252.5)\end{array}$ & $\begin{array}{l}813,217.3(727,039.6- \\
960,672.8)\end{array}$ & $43.7(37.3-56.4)$ & $31.6(28.2-37.2)$ & $-27.8(-40.3$ to -14.2$)$ \\
\hline Low-middle SDI & $\begin{array}{l}549,795.0(462,013.9- \\
726,880.2)\end{array}$ & $\begin{array}{l}919,232.5(751,488.8- \\
1,066,251.6)\end{array}$ & $68.4(57.0-88.3)$ & $58.0(47.3-66.8)$ & $-15.3(-32.5$ to 8.6$)$ \\
\hline Low SDI & $\begin{array}{l}244,325.0(185,104.9- \\
330,618.6)\end{array}$ & $459,380.2(367,333.8-$ & $75.1(55.2-00.8)$ & $64.0(50.8-81.3)$ & $-14.9(-29.9$ to 3.3$)$ \\
\hline \multicolumn{6}{|l|}{ Region } \\
\hline Andean Latin America & $41,681.2(49,404.7-30,752.3)$ & $43,654.9(34,042.4-57,110.3)$ & $144.8(108.3-173.2)$ & $72.5(56.6-96.0)$ & $-49.9(-63.0$ to -25.6$)$ \\
\hline Australasia & $5562.6(6030.6-5108.7)$ & $7137.4(6312.5-8100.2)$ & $24.4(22.4-26.5)$ & $16.6(14.7-18.8)$ & $-32.2(-39.4 .2$ to -21.6$)$ \\
\hline Caribbean & $13,236.4(15,634.5-11,659.6)$ & $20,254.6(16,880.2-24,620.4)$ & $44.5(39.3-51.6)$ & $40.1(33.3-48.7)$ & $-9.9(-23.8 .0$ to 5.7$)$ \\
\hline Central Asia & $41,215.8(45,305.5-36,642.2)$ & $60,311.2(51,431.9-69,088.5)$ & $77.1(68.3-85.3)$ & $67.4(57-76.9)$ & $-12.5(-24.4$ to 1.0$)$ \\
\hline Central Europe & $\begin{array}{l}147,739.5(161,428.9- \\
141,054.8)\end{array}$ & $140,578.0(123,451.4-$ & $106.0(101.2-115.9)$ & $84.4(74-95.6)$ & $-20.4(-30.9 .0$ to -9.5$)$ \\
\hline Central Latin America & $80,017.9(84,085.9-74,360)$ & $\begin{array}{l}141,733.4(121,885.5- \\
165,140.4)\end{array}$ & $67.0(63.3-70.8)$ & $56.5(48.7-65.7)$ & $-15.7(-27.4 .0$ to -0.9$)$ \\
\hline Central Sub-Saharan Africa & $17,413.0(29,045.2-11,986.3)$ & $35,823.5(21,454.6-62,656.8)$ & $54.4(36.7-90.9)$ & $44.6(26.5-80.0)$ & $-18.0(-42.1 .1$ to 10.6$)$ \\
\hline East Asia & $\begin{array}{l}333,276.6(431,225.3- \\
266,027.9)\end{array}$ & $319,973(255,865.5-$ & $31.7(25.7-41.5)$ & $16.5(13.2-19.6)$ & $-47.9(-62.1 .3$ to -32.1$)$ \\
\hline Eastern Europe & $\begin{array}{l}283,386.3(383,366.6- \\
254,907.8)\end{array}$ & $\begin{array}{l}558,129.2(481,178.6- \\
640,436.3)\end{array}$ & $109.2(98.2-146.8)$ & $206.7(178.3-237.9)$ & $-89.2(-29.5 .2$ to 120.3$)$ \\
\hline Eastern Sub-Saharan Africa & $51,548.1(78,409.9-34,736.6)$ & $\begin{array}{l}103,187.0(65,327.8- \\
170,638.8)\end{array}$ & $49.6(32.1-74.6)$ & $43.3(27.1-73.7)$ & $-12.7(-34.4 .1$ to 14.2$)$ \\
\hline High-income Asia Pacific & $54,433.0(66,219.4-47,665.2)$ & $48,064.3(41,966.4-56,862.1)$ & $27.6(24.1-33.4)$ & $14.7(12.7-17.4)$ & $-46.6(-57.3 .3$ to -37$)$ \\
\hline $\begin{array}{l}\text { High-income North } \\
\text { America }\end{array}$ & $\begin{array}{l}100,034.4(109,713.5- \\
92,703.1)\end{array}$ & $145,495.4(134,413.4-$ & $30.8(28.5-33.9)$ & $28.6(26.4-31.6)$ & $-7.4(-10.7 .0$ to -1.9$)$ \\
\hline $\begin{array}{l}\text { North Africa and Middle } \\
\text { East }\end{array}$ & $50,486.0(69,094.5-42,546.5)$ & $\begin{array}{l}91,833.5(74,033.1- \\
111,078.4)\end{array}$ & $25.8(21.5-35.0)$ & $19.5(15.7-23.3)$ & $-24.6(-41.2 .0$ to -4.6$)$ \\
\hline Oceania & $1540.1(2186.2-1100.1)$ & $3181.1(2194.1-4459.1)$ & $33.9(23.9-47.8)$ & $30.3(21.4-42.0)$ & $-10.7(-32.7 .1$ to 16.6$)$ \\
\hline South Asia & $\begin{array}{l}548,424.8(759,731.1- \\
448,431.7)\end{array}$ & $\begin{array}{l}909,993.5(707,700.0- \\
1,093,715.6)\end{array}$ & $69.9(56.5-95.3)$ & $55.3(43.1-66.4)$ & $-20.9(-43.6 .0$ to 8.2$)$ \\
\hline Southeast Asia & $187,984.2(286,963-145,388)$ & $\begin{array}{l}254,592.2(204,677.0- \\
376,978.7)\end{array}$ & $54.5(42.3-81.1)$ & $37.9(30.8-54.8)$ & $-30.5(-43.6 .1$ to -12.7$)$ \\
\hline Southern Latin America & $37,055.6(40,031.4-33,771.4)$ & $40,292.4(36,539.8-45,858.7)$ & $78.6(71.7-85.0)$ & $51.6(46.8-58.8)$ & $-34.3(-42.4 .2$ to -21.1$)$ \\
\hline $\begin{array}{l}\text { Southern Sub-Saharan } \\
\text { Africa }\end{array}$ & $12,983.4(16,365.4-10,471.3)$ & $21,220.6(16,850.1-25,384.5)$ & $34.6(28.1-43.7)$ & $30.0(23.8-35.7)$ & $-13.3(-38.3 .0$ to 5.8$)$ \\
\hline Tropical Latin America & $90,244.6(95,308.1-85,172.8)$ & $\begin{array}{l}175,860.2(155,755.4- \\
189,220.5)\end{array}$ & $75.4(71.0-79.9)$ & $70.6(62.5-76.0)$ & $-6.3(-17.3 .0$ to 2.0$)$ \\
\hline Western Europe & $\begin{array}{l}196,013.5(212,945.9- \\
184,807.6)\end{array}$ & $\begin{array}{l}199,700.1(183,864.2- \\
224,326.9)\end{array}$ & $38.8(36.6-41.9)$ & $27.1(25.2-30.4)$ & $-30.1(-35.2 .2$ to -24.1$)$ \\
\hline $\begin{array}{l}\text { Western Sub-Saharan } \\
\text { Africa }\end{array}$ & $\begin{array}{l}143,538.8(213,834.4- \\
95,218.6)\end{array}$ & $\begin{array}{l}320,090.3(226,679.1- \\
458,546.1)\end{array}$ & $122.5(80.9-189.4)$ & $115.5(82.3-165.9)$ & $-5.7(-33.1$ to 26.2$)$ \\
\hline
\end{tabular}

EAPC, estimated annual percentage change; UI, uncertainty interval; DALY, disability-adjusted life-year 
expected values based on SDI for nearly all years between 1990 and 2019.

\section{The burden of acute pancreatitis and age structure}

We analyzed the incidence rate in five age groups: under 5 years, 5-14 years, 15-49 years, 50-69 years, and over 70 years in the globe and different SDI regions. The results demonstrated that the incidence rate (per $100,000)$ in people above 70 years was the highest across all regions from 1990 to 2019 (Additional file 1: Fig. S1). Based on data of 1990 and 2019, all the incidence, mortality and DALY rates rose with advancing age; of note, the mortality rate rose sharply in patients aged 70 years and older (Additional file 1: Fig. S2).

The mortality of acute pancreatitis due to alcohol etiology In 1990, there were 0.8 males died per 100,000 due to alcohol-related pancreatitis in the globe, accounted for $36.1 \%(0.8 / 2.1)$ of all cause pancreatitis-related ASMR; among females, this percentage was $15.4 \%(0.2 / 1.3)$ (Additional file 1: Fig. S3a). The attributable fraction of alcohol etiology on mortality was highest among people in the high SDI region for both males and females.

Up to 2019, alcohol etiology still responsible for a sizable fraction of mortality rate in males than that in females $(40.2 \%$ vs $12.3 \%)$ globally, and was the dominant cause for acute pancreatitis' ASMR in males in the high (50.2\%) and high-middle SDI (52.4\%) regions (Additional file 1: Fig. S3b). The five highest alcohol etiology caused ASMR were seen in Russia $(2.7 / 100,000)$, Ukraine $(2.3 / 100,000)$, Republic of Moldova $(2.3 / 100,000)$, Belarus $(2.2 / 100,000)$ and Lithuania $(2.1 / 100,000)$ (Additional file 1: Fig. S4).

\section{Discussion}

This study provides a systematic analysis of acute pancreatitis' incidence, mortality, DALY and corresponding trends across all 204 countries and territories over a 30-year period. We estimated that in 2019, 2,814,972.3 acute pancreatitis occurred, with 115,053.2 (4.1\%) person died globally. Between 1990 and 2019, the age-standardized incidence of acute pancreatitis declined in most countries, but the condition persisted severe in some of the regions, especially in Eastern Europe and Highincome North America.

Our global estimate of acute pancreatitis' incidence in 2019 is close to the estimation in previous meta-analysis $(33.7 / 100,000)$; in addition, the GBD estimation of 1.4 deaths per 100,000 is also similar to the meta-analytical result $(1.6$ deaths per 100,000) [7]. Of note, the previous analyses failed to make the combined best use of different types of data on incidence, mortality, DALY, and risk factors reported in literature sources, claims databases, and vital registration systems. GBD estimates the attributable disease burden using a comparative risk assessment strategy through all countries and territories around the world [26]. The global analysis and cross-region comparisons could improve the comprehensive understanding of the burden of acute pancreatitis.

Although our results revealed an increase in acute pancreatitis' incident cases during the 30-year study period, the mortality rate declined continuously. We have also observed a decreasing mortality rate over the last decade $[8,27-30]$. These outcomes are justifiable, for patients with acute pancreatitis are becoming easier to identify with better testing approaches, and the complications can be detected at an earlier stage in the disease course [31]. Furthermore, improvements on the treatment of severe cases in intensive care units, combined with multidisciplinary management strategies have all contributed to the decrease in the acute pancreatitis-related death $[32,33]$.

However, it should be pointed out that patients in low SDI region had more than twofold higher mortality of acute pancreatitis than people in the high SDI region.
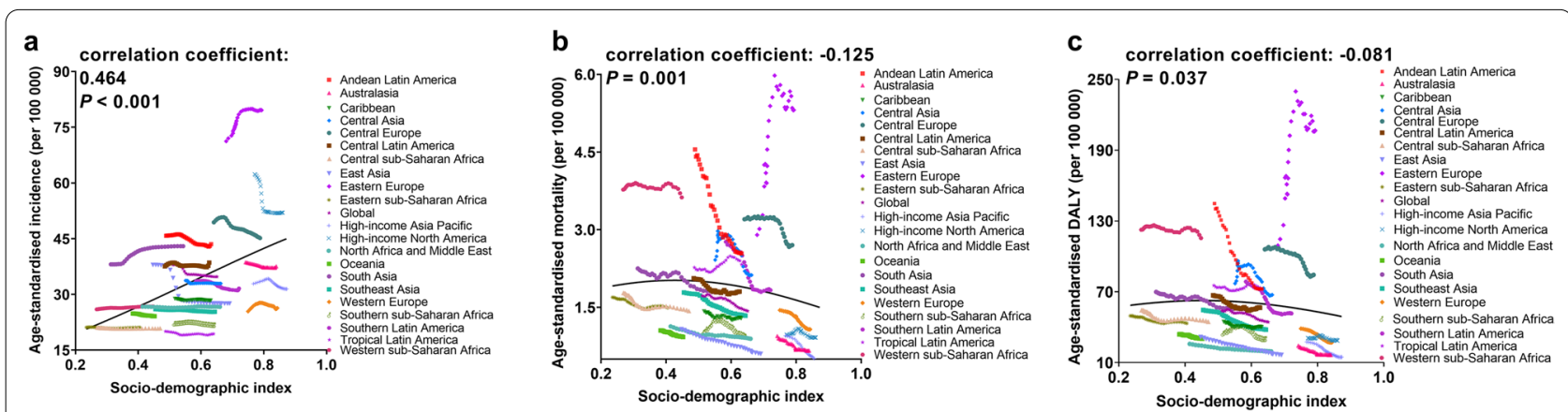

Fig. 3 The change trends and correlation analyses of acute pancreatitis' burden and SDI for 21 world regions from 1990 to 2019. The age-standardized incidence $(\mathbf{a})$, mortality $(\mathbf{b})$ and DALY (c) rates per 100,000 person-years is shown. The solid black line is a mixed-effects and spatiotemporal Gaussian process regression, and represents the expected values across the spectrum of the SDI. DALY: disability-adjusted life-year; SDI: socio-demographic index 
Socioeconomic factors are important variables in acute pancreatitis' epidemiology, especially for the mortality. There were indications that the size of hospital was closely associated with the risk of acute pancreatitis-related fatality. Compared with small hospitals, patients admitted into the large hospitals had a lower risk of death [34-36]. Better outcomes have also been observed for hospitals and surgeons with high volumes of cases [37, 38]. By contrast, the prognosis of acute pancreatitis in low-income regions or socially deprived areas was poorer [39, 40]. The morbidity and mortality of acute pancreatitis would be expected to correlate with the national health system infrastructure as regards the existence or not of specialist tertiary pancreatitis units, and improved outcomes might be achieved in countries with improved access to clinical resources such as specialist tertiary pancreatitis services.

Differences also existed between different age groups. We found that the incidence rate was significantly higher in population over 70 years old. Actually, aging is an important factor that contributing to acute pancreatitis. The incidence of acute pancreatitis attributable to gallstone increased sharply with age for both men and women [40]. In addition, Floyd et al. [13] found that the increase in the drug consumption, such as azathioprine, was also correlated with the higher incidence of acute pancreatitis in the elderly. Moreover, strong association between old age and mortality rate was also reported previously [13], and this was in line with our finding that mortality rate increased with aging and rose sharply in those aged 70 years and older. This is to be expected in relation to the more numerous and more severe coexisting conditions in the elderly people [41, 42].

In GBD 2019, we found alcohol etiology was an important risk factor for acute pancreatitis-related death. Males were much more likely to suffer from fatality due to alcohol-induced acute pancreatitis. In pace with the drinking prevalence increased towards higher levels of SDI [43], the fraction of mortality attributed to alcoholic acute pancreatitis also had a mushrooming rise towards high SDI region for both men and women. Notably, there were evidence indicated that the type of alcohol consumption would affect the acute pancreatitis' risk. Roberts et al. [40] revealed that alcoholic acute pancreatitis was positively correlated with spirits and beer, but negatively with wine. Other studies showed that the risk of acute pancreatitis had a dose-response association with the number of units of spirits consumption, but not associated with beer or wine [44]. Therefore, public health policies that focus on reducing population-level alcohol intake might be effective in reducing the morbidity and mortality of acute pancreatitis.

This study has some limitations. First, apart from alcohol etiology, gallstone is another major cause of acute pancreatitis in most countries [45], so are the other less common but meaningful causes such as hypertriglyceridemia. However, the quantitative effect of gallstone and other causes on acute pancreatitis could not be assessed owing to unavailable data in the current round of GBD. Second, in order to formulate more effective preventive measures, proper stratification of severity grades and analysis for subtypes of acute pancreatitis according to aetiology is necessary. However, based on the data of GBD 2019, we couldn't obtain such information. In addition, GBD faces several challenges for estimating cause-specific non-fatal and fatal burden of acute pancreatitis. Even though it employs spatiotemporal methods to inform estimates for locations with sparse data, these strategies cannot completely resolve issues when data are not available for some regions. As vital statistic systems and civil registration provide important information for disease preventions and public health policies, strengthening of these systems is essential for public health. An ongoing effort of adding new sources of data to the GBD should be taken to overcome the limitations.

\section{Conclusions}

In summary, the age-standardized incidence, mortality and DALY rates of acute pancreatitis decreased gradually in the globe. However, the overall burden remains high with aging population, and will not decrease without effective strategies to address associated risk factors. The attributable burden is higher in males and elderly, and alcohol etiology is an important driver that contribute to the morbidity and mortality. There were substantial differences in the burden of acute pancreatitis across regions, geographically targeted considerations are needed to tailor future interventions to relieve the burden of acute pancreatitis in specific countries, especially for Eastern Europe.

\begin{abstract}
Abbreviations
CODEm: Cause of death ensemble model; DALYs: Disability-adjusted life year; GBD: Global burden of diseases; EAPC: Estimated annual percentage change; ASIR: Age-standardized incidence rate; ASMR: Age-standardized mortality rate; GHDx: Global health data exchange; ICD: International classification of diseases; SDI: Socio-demographic Index; ST-GPR: Spatiotemporal Gaussian process regression; Uls: Uncertainty intervals; YLDs: Years lived with disability; YLLs: Years of life lost.
\end{abstract}

\section{Supplementary Information}

The online version contains supplementary material available at https://doi. org/10.1186/s12876-021-01906-2.

Additional file 1. Supplementary methods, tables, and figures. 


\section{Acknowledgements}

We appreciate the works by the Global Burden of Disease study 2019 collaborators. We would like to thank American Journal Experts (https://www.aje. com) for editing this manuscript.

\section{Authors' contributions}

$\mathrm{CL}$ and $\mathrm{MJ}$ conceptualized the study. MJ devised the design of the study. CP, $J \mathrm{~L}$ and $\mathrm{LX}$ collected the data. $\mathrm{CL}$ and $\mathrm{MJ}$ contributed to the data analysing. MJ wrote the first draft of the manuscript. CP, JL and LX edited the paper. $\mathrm{CL}$ revised the manuscript. All authors approved the final version of the manuscript.

\section{Funding}

This work was supported by the Project funded by China Postdoctoral Science Foundation (2020M682422).

\section{Availability of data and materials}

The datasets generated for this study can be found in the GBD at http://ghdx. healthdata.org/gbd-results-tool.

\section{Declarations}

Ethics approval and consent to participate

This study did not require ethical approval.

\section{Consent for publication}

Not applicable.

\section{Competing interests}

The authors declare that they have no competing interests.

\section{Author details}

${ }^{1}$ Department of Geratology, Hubei Provincial Hospital of Integrated Chinese and Western Medicine, Wuhan 430015, China. ${ }^{2}$ Department of Medical Ultrasound, Tongji Hospital, Tongji Medical College, Huazhong University of Science and Technology, Wuhan 430030, China. ${ }^{3}$ Department of Emergency Medicine, Nanfang Hospital, Southern Medical University, Guangzhou 510515, China. ${ }^{4}$ Department of Critical Care Medicine, Wuhan Central Hospital, Tongji Medical College, Huazhong University of Science and Technology, Wuhan 430000, China.

Received: 11 May 2021 Accepted: 12 August 2021

Published online: 25 August 2021

\section{References}

1. Nøjgaard C. Prognosis of acute and chronic pancreatitis-a 30-year follow-up of a Danish cohort. Dan Med Bull. 2010;57:B4228.

2. Ekbom A, McLaughlin JK, Karlsson BM, Nyrén O, Gridley G, Adami HO, et al. Pancreatitis and pancreatic cancer: a population-based study. J Natl Cancer Inst. 1994;86:625-7.

3. Peery AF, Crockett SD, Barritt AS, Dellon ES, Eluri S, Gangarosa LM, et al. Burden of gastrointestinal, liver, and pancreatic diseases in the United States. Gastroenterology. 2015;149:1731-41.

4. Schepers NJ, Bakker OJ, Besselink MG, Ahmed AU, Bollen TL, Gooszen HG, et al. Impact of characteristics of organ failure and infected necrosis on mortality in necrotising pancreatitis. Gut. 2019;68:1044-51.

5. van Santvoort HC, Bakker OJ, Bollen TL, Besselink MG, Ahmed AU, Schrijver AM, et al. A conservative and minimally invasive approach to necrotizing pancreatitis improves outcome. Gastroenterology. 2011;141:1254-63.

6. Bang JY, Wilcox CM, Arnoletti JP, Varadarajulu S. Superiority of endoscopic interventions over minimally invasive surgery for infected necrotizing pancreatitis: meta-analysis of randomized trials. Dig Endosc. 2020;32:298-308.

7. Xiao AY, Tan ML, Wu LM, Asrani VM, Windsor JA, Yadav D, et al. Global incidence and mortality of pancreatic diseases: a systematic review, meta-analysis, and meta-regression of population-based cohort studies. Lancet Gastroenterol Hepatol. 2016;1:45-55.
8. Bilal M, Kline KT, Trieu JA, Saraireh H, Desai M, Parupudi S, et al. Trends in same-admission cholecystectomy and endoscopic retrograde cholangiopancreatography for acute gallstone pancreatitis: a nationwide analysis across a decade. Pancreatology. 2019;19:524-30.

9. Krishna SG, Kamboj AK, Hart PA, Hinton A, Conwell DL. The changing epidemiology of acute pancreatitis hospitalizations. Pancreas. 2017;46:482-8.

10. Roberts SE, Thorne K, Evans PA, Akbari A, Samuel DG, Williams JG. Mortality following acute pancreatitis: social deprivation, hospital size and time of admission: record linkage study. BMC Gastroenterol. 2014;14:153.

11. Chung S, Chen K, Xirasagar S, Tsai M, Lin H. More than 9-times increased risk for pancreatic cancer among patients with acute pancreatitis in Chinese population. Pancreas. 2012;41:142-6.

12. Sadr-Azodi O, Andrén-Sandberg Å, Orsini N, Wolk A. Cigarette smoking, smoking cessation and acute pancreatitis: a prospective populationbased study. Gut. 2011;61:262-7.

13. Floyd A, Pederson L, Nielsen GL, Thorlacius-Ussing O, Sorensen HT. Secular trends in incidence and 30-day case fatality of acute pancreatitis in North Jutland County, Denmark: a register-based study from 1981-2000. Scand J Gastroenterol. 2009;37:1461-5.

14. Blomgren KJ, Sundström A, Steineck G, Wiholm B. Interviewer variability —quality aspects in a case-control study. Eur J Epidemiol. 2006;21:267-77.

15. Lindkvist B, Appelros S, Manjer J, Borgström A. Trends in incidence of acute pancreatitis in a Swedish population: is there really an increase? Clin Gastroenterol H. 2004;2:831.

16. Kyu HH, Abate D, Abate KH, Abay SM, Abbafati C, Abbasi N, et al. Global, regional, and national disability-adjusted life-years (DALYS) for 359 diseases and injuries and healthy life expectancy (HALE) for 195 countries and territories, 1990-2017: a systematic analysis for the Global Burden of Disease Study 2017. Lancet. 2018;392:1859-922.

17. James SL, Abate D, Abate KH, Abay SM, Abbafati C, Abbasi N, et al. Global, regional, and national incidence, prevalence, and years lived with disability for 354 diseases and injuries for 195 countries and territories, 1990-2017: a systematic analysis for the Global Burden of Disease Study 2017. Lancet. 2018:392:1789-858.

18. Watkins DA, Johnson CO, Colquhoun SM, Karthikeyan G, Beaton A, Bukhman G, et al. Global, regional, and national burden of rheumatic heart disease, 1990-2015. N Engl J Med. 2017;377:713-22.

19. Global, regional, and national age-sex-specific mortality for 282 causes of death in 195 countries and territories, 1980-2017: a systematic analysis for the Global Burden of Disease Study 2017. Lancet. 2018; 392:1736-88.

20. Ouyang G, Pan G, Liu Q, Wu Y, Liu Z, Lu W, et al. The global, regional, and national burden of pancreatitis in 195 countries and territories, 1990-2017: a systematic analysis for the Global Burden of Disease Study 2017. BMC Med. 2020; 18

21. Murray CJL, Aravkin AY, Zheng P, Abbafati C, Abbas KM, Abbasi-Kangevari M, et al. Global burden of 87 risk factors in 204 countries and territories, 1990-2019: a systematic analysis for the Global Burden of Disease Study 2019. Lancet. 2020;396:1223-49.

22. Vos T, Abbasi M, Abbasifard M, Abbastabar H, Abd-Allah F, Abdelalim A, et al. Global burden of 369 diseases and injuries in 204 countries and territories, 1990-2019: a systematic analysis for the Global Burden of Disease Study 2019. Lancet. 2020;396:1204-22.

23. Collaborators GPAF. Population and fertility by age and sex for 195 countries and territories, 1950-2017: a systematic analysis for the Global Burden of Disease Study 2017. Lancet. 2018;392:1995-2051.

24. Li X, Cao X, Guo M, Xie M, Liu X. Trends and risk factors of mortality and disability adjusted life years for chronic respiratory diseases from 1990 to 2017: systematic analysis for the Global Burden of Disease Study 2017. BMJ. 2020; 368:m234

25. Stevens GA, Alkema L, Black RE, Boerma JT, Collins GS, Ezzati M, et al. Guidelines for accurate and transparent health estimates reporting: the GATHER statement. Lancet. 2016;388:e19-23.

26. Petrov MS, Yadav D. Global epidemiology and holistic prevention of pancreatitis. Nat Rev Gastroenterol Hepatol. 2019;16:175-84.

27. McNabb-Baltar J, Ravi P, Isabwe GA, Suleiman SL, Yaghoobi M, Trinh QD, et al. A population-based assessment of the burden of acute pancreatitis in the United States. Pancreas. 2014;43:687-91.

28. Fagenholz PJ, Castillo CF, Harris NS, Pelletier AJ, Camargo CJ. Increasing United States hospital admissions for acute pancreatitis, 1988-2003. Ann Epidemiol. 2007;17:491-7. 
29. Singla A, Simons J, Li Y, Csikesz NG, Ng SC, Tseng JF, et al. Admission volume determines outcome for patients with acute pancreatitis. Gastroenterology. 2009;137:1995-2001.

30. Brown A, Young B, Morton J, Behrns K. N Shaheen (2008) Are health related outcomes in acute pancreatitis improving? An analysis of national trends in the US from 1997 to 2003. JOP. 2008;9:408-14.

31. Boxhoorn L, Voermans RP, Bouwense SA, Bruno MJ, Verdonk RC, Boermeester MA, et al. Acute pancreatitis. Lancet. 2020;396:726-34.

32. McKay CJ, Evans S, Sinclair M, Carter CR, Imrie CW. High early mortality rate from acute pancreatitis in Scotland, 1984-1995. Br J Surg. 1999;86:1302-5.

33. Mann DV, Hershman MJ, Hittinger R, Glazer G. Multicentre audit of death from acute pancreatitis. Br J Surg. 1994;81:890-3.

34. Andrén-Sandberg A. Organization of care for pancreatic cancer. N Am J Med Sci. 2011;3:400-5.

35. Murata A, Matsuda S, Mayumi T, Yokoe M, Kuwabara K, Ichimiya Y, et al. Effect of hospital volume on clinical outcome in patients with acute pancreatitis, based on a national administrative database. Pancreas. 2011;40:1018-23.

36. Shen $H N$, Lu CL, Li CY. The effect of hospital volume on patient outcomes in severe acute pancreatitis. BMC Gastroenterol. 2012;12:112.

37. Chowdhury MM, Dagash $H$, Pierro A. A systematic review of the impact of volume of surgery and specialization on patient outcome. Br J Surg. 2007;94:145-61.

38. McAteer JP, LaRiviere CA, Drugas GT, Abdullah F, Oldham KT, Goldin AB. Influence of surgeon experience, hospital volume, and specialty designation on outcomes in pediatric surgery: a systematic review. JAMA Pediatr. 2013;167:468-75.
39. Roberts SE, Williams JG, Meddings D, Goldacre MJ. Incidence and case fatality for acute pancreatitis in England: geographical variation, social deprivation, alcohol consumption and aetiology - a record linkage study. Aliment Pharmacol Ther. 2008;28:931-41.

40. Roberts SE, Akbari A, Thorne K, Atkinson M, Evans PA. The incidence of acute pancreatitis: impact of social deprivation, alcohol consumption, seasonal and demographic factors. Aliment Pharmacol Ther. 2013;38:539-48.

41. Hong S, Qiwen B, Ying J, Wei A, Chaoyang T. Body mass index and the risk and prognosis of acute pancreatitis: a meta-analysis. Eur J Gastroenterol Hepatol. 2011;23:1136-43.

42. Krishna SG, Hinton A, Oza V, Hart PA, Swei E, El-Dika S, et al. Morbid obesity is associated with adverse clinical outcomes in acute pancreatitis: a propensity-matched study. Am J Gastroenterol. 2015;110:1608-19.

43. Griswold MG, Fullman N, Hawley C, Arian N, Zimsen SRM, Tymeson HD, et al. Alcohol use and burden for 195 countries and territories, 1990-2016: a systematic analysis for the Global Burden of Disease Study 2016. Lancet. 2018;392:1015-35.

44. Schmidt DN. Apparent risk factors for chronic and acute pancreatitis in Stockholm county: spirits but not wine and beer. Int J Pancreatol. 1991;8:45-50.

45. Roberts SE, Morrison-Rees S, John A, Williams JG, Brown TH, Samuel DG. The incidence and aetiology of acute pancreatitis across Europe. Pancreatology. 2017;17:155-65.

\section{Publisher's Note}

Springer Nature remains neutral with regard to jurisdictional claims in published maps and institutional affiliations.
Ready to submit your research? Choose BMC and benefit from:

- fast, convenient online submission

- thorough peer review by experienced researchers in your field

- rapid publication on acceptance

- support for research data, including large and complex data types

- gold Open Access which fosters wider collaboration and increased citations

- maximum visibility for your research: over $100 \mathrm{M}$ website views per year

At BMC, research is always in progress.

Learn more biomedcentral.com/submissions 